\title{
Structure and Optical Properties of Some Layered Two-Dimensional Transition- Metal Dichalcogenides: Molybdenum Disulfide, Molybdenum Diselenide, and Tungsten Diselenide
}

\author{
E.S. Reifler ${ }^{1}$, N.T. Nuhfer ${ }^{1}$, and E. Towe ${ }^{1,2}$ \\ 1Department of Materials Science and Engineering, Carnegie Mellon University, Pittsburgh, PA, USA \\ 2Department of Electrical and Computer Engineering, Carnegie Mellon University, Pittsburgh, PA, USA
}

This paper presents a systematic study of intrinsic characteristics of layered two-dimensional transitionmetal dichalcogenides, with a focus on molybdenum disulfide, molybdenum diselenide, and tungsten diselenide. Recent advances in high-resolution transmission electron microscopy (HRTEM) have made atomic-resolution imaging possible, thus enabling one to closely examine real and reciprocal space structures of two-dimensional materials. Our HRTEM micrographs, for example, confirm the expected hexagonal structure of the materials. Furthermore, lattice constant values with resolution on the order of tenths of angstroms could be extracted from the micrographs. Raman and photoluminescence spectroscopies are used to investigate the optical characteristics of the materials. These measurements provide insight into the properties of this class of materials, and their potential use in promising applications in electronic and photonic devices [1-5].

The samples for the high-resolution transmission electron microscopy were prepared directly from natural bulk single crystal molybdenum disulfide and from synthetic bulk molybdenum diselenide and tungsten diselenide crystals. This direct sample preparation method minimizes introduction of defects that might be formed by transfer of the samples from a separate substrate to a TEM grid. The instrument used in our imaging work was an image-aberration-corrected FEI Titan G2 80-300 transmission electron microscope; this system is capable of providing atomic resolution micrographs. Bulk and monolayer HRTEM images of molybdenum disulfide and tungsten diselenide are shown in Figure 1. The direct hexagonal patterns visually evident in the images and extractable from a Fourier analysis of the images confirm the expected hexagonal structure of the real and reciprocal space lattices of these materials. We were able to identify single-layer areas based on the relative intensities of neighboring atomic columns [6]. The lattice constants of the bulk and monolayer molybdenum disulfide, molybdenum diselenide, and tungsten diselenide were extracted by analyzing the HRTEM images; the lattice constants extracted from these analyses compare well with theoretical values in the literature.

To investigate the optical characteristics of the two-dimensional transition-metal dichalcogenides, monolayer and few-layer samples were mechanically exfoliated from the bulk crystals [7-8]. Raman spectroscopy and photoluminescence spectroscopy measurements were performed using an integrated Renishaw Raman-photoluminescence system equipped with a 532-nm laser excitation source; this system is capable of a resolution of $\sim 1 \mathrm{~cm}^{-1}$ in the Raman mode and of $\sim 0.3 \mathrm{meV}$ in the photoluminescence mode. By monitoring the spectral location of the active Raman modes, it was possible to determine the number of layers present in a few-layer sample of a dichalcogenide [9]. This determination was corroborated by the spectral location of the photoluminescence emission peak. Our experiments show that photoluminescence emission from a transition-metal dichalcogenide monolayer is significantly more intense than emission from few-layer or bulk samples; this is shown in Figure 2. The higher intensity of the emission from a monolayer is characteristic of a direct band gap 
semiconductor. Relatively lower emission intensities from thicker samples are indicative of indirect band gap materials.

Together, these experimental measurements provide a useful data set for understanding the properties of two-dimensional transition-metal dichalcogenides. When augmented with theoretical considerations, they provide a basis on which to build an exploration of the potential applications of these materials.

\section{References:}

[1] Hsiao-Yu Chang, et al., ACS Nano 7, No. 6 (2013), pp. 5446-52.

[2] Jongwon Yoon, et al., Small 9, No. 19 (2013), pp. 3295-3300.

[3] Simone Bertolazzi, Jacopo Brivio, and Andras Kis, ACS Nano, Vol. 5, No. 12 (2011), pp. 9703-09.

[4] Di Xiao, et al., Phys Rev Lett. 108 (2012), 196802.

[5] B. Radisavljevic, et al., Nature Nanotechnology, 6 (2011), pp.147-150.

[6] Jonathan N. Coleman, et al., Science 331 (2011), pp. 568-571.

[7] K.S. Novoselov, et al., Science 306 (2004), pp. 666-669.

[8] K.S. Novoselov, et al., PNAS 102, No. 30 (2005), pp. 10451-10453.

[9] Changgu Lee, et al., ACS Nano 4, No. 5 (2010), pp. 2695-2700.
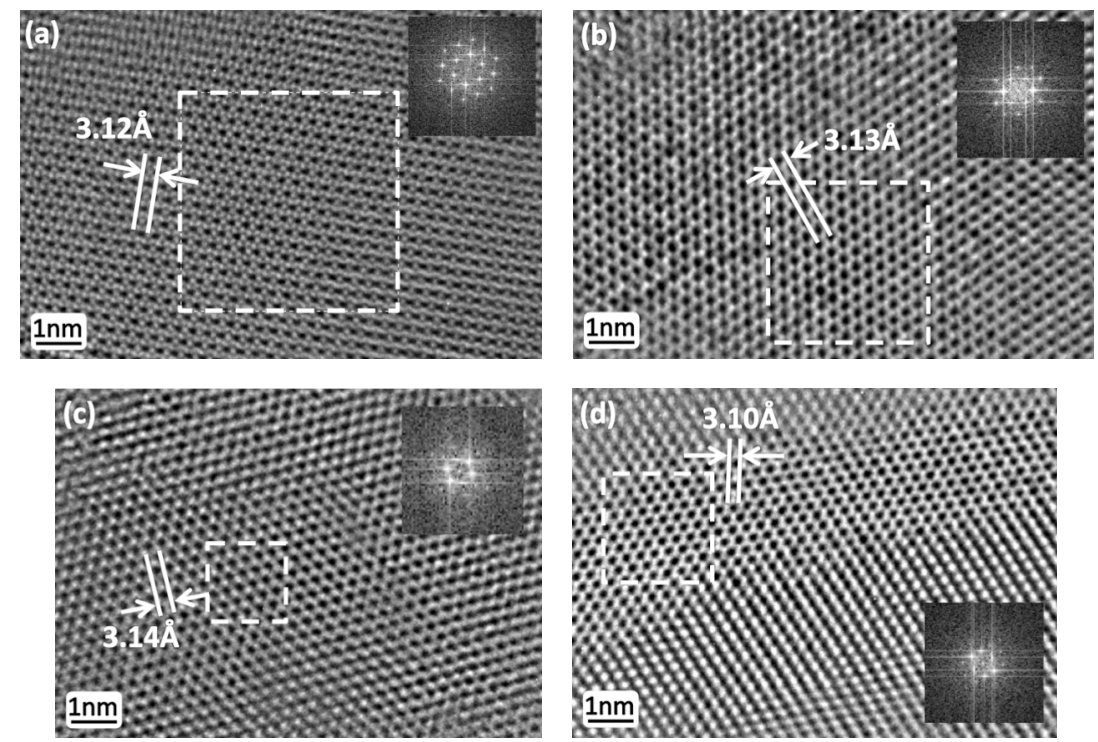

Figure 1. HRTEM images of (a) monolayer molybdenum disulfide, (b) bulk molybdenum disulfide, (c) monolayer tungsten diselenide, and (d) bulk tungsten diselenide. The insets are fast Fourier transforms of the areas outlined by the white dashed boxes. Lattice constant measurements are indicated in each image. All scale bars are $1 \mathrm{~nm}$.
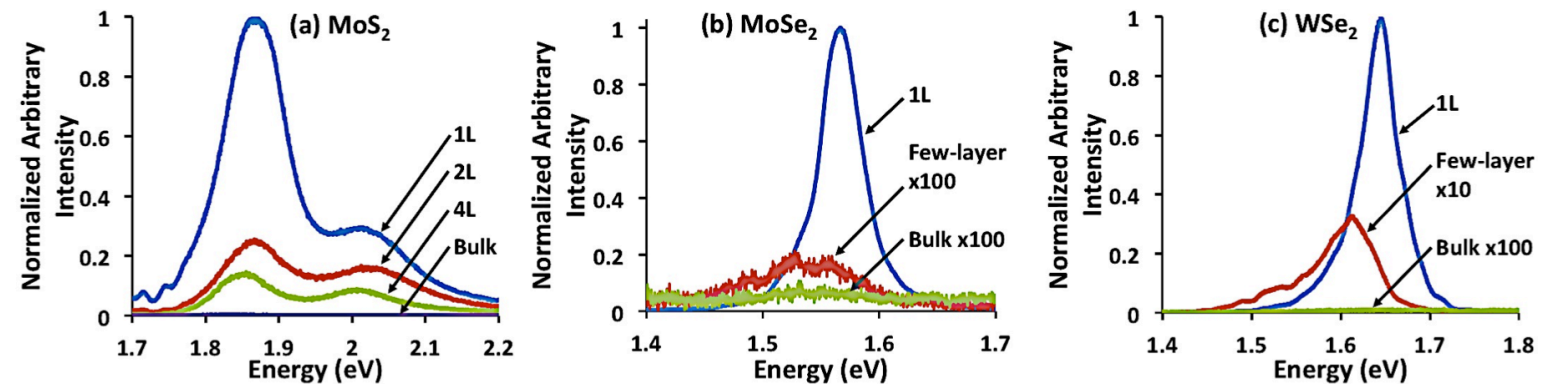

Figure 2. Photoluminescence spectra of monolayer, few-layer, and bulk (a) molybdenum disulfide, (b) molybdenum diselenide, and (c) tungsten diselenide. 